\title{
EEG hyper-connectivity in high-risk infants is associated with later autism
}

\author{
Elena V Orekhova ${ }^{1 *}$, Mayada Elsabbagh², Emily JH Jones', Geraldine Dawson ${ }^{3}$, Tony Charman ${ }^{4}$, \\ Mark H Johnson ${ }^{1}$ and The BASIS Team
}

\begin{abstract}
Background: It has been previously reported that structural and functional brain connectivity in individuals with autism spectrum disorders (ASD) is atypical and may vary with age. However, to date, no measures of functional connectivity measured within the first 2 years have specifically associated with a later ASD diagnosis.

Methods: In the present study, we analyzed functional brain connectivity in 14-month-old infants at high and low familial risk for ASD using electroencephalography (EEG). EEG was recorded while infants attended to videos. Connectivity was assessed using debiased weighted phase lag index (dbWPLI). At 36 months, the high-risk infants were assessed for symptoms of ASD.

Results: As a group, high-risk infants who were later diagnosed with ASD demonstrated elevated phase-lagged alpha-range connectivity as compared to both low-risk infants and high-risk infants who did not go on to ASD. Hyper-connectivity was most prominent over frontal and central areas. The degree of hyper-connectivity at 14 months strongly correlated with the severity of restricted and repetitive behaviors in participants with ASD at 3 years. These effects were not attributable to differences in behavior during the EEG session or to differences in spectral power.
\end{abstract}

Conclusions: The results suggest that early hyper-connectivity in the alpha frequency range is an important feature of the ASD neurophysiological phenotype.

Keywords: Autism spectrum disorders, EEG, Connectivity, Alpha, Infants, Siblings

\section{Background}

Autism spectrum disorder (ASD) is primarily characterized by impairments in social communication skills and the presence of repetitive and stereotypical behaviors [1]. Recent research shows that many genes implicated in ASD are involved in formation and regulation of synaptic pathways and neural connections [2,3]. Given that the pruning and modification of such synapses and neural pathways are partly shaped by experience $[4,5]$, studies of brain connectivity in very young children with ASD, or in infants 'at risk' for developing ASD, are critical.

Structural and functional MRI studies in adults with ASD have predominantly reported weaker long-range

\footnotetext{
* Correspondence: orekhova.elena.v@gmail.com

${ }^{1}$ Centre for Brain and Cognitive Development, School of Psychology,

Birkbeck, University of London, Henry Welcome Building, London WC1E 7HX, UK

Full list of author information is available at the end of the article
}

cortico-cortical connections [6,7]. The studies that used electro- (EEG) and magneto-encephalography (MEG) also primarily found reduced functional connectivity, although the results were more variable (see Additional file 1). In contrast, more recent fMRI studies in younger (pre-pubertal) children with ASD have reported increased functional connectivity in brain networks [8-10]. Further, functional hyper-connectivity associates with greater severity of autism symptoms in children [8]. In line with the increased functional connectivity findings, recent diffusion tensor imaging studies revealed that young children and toddlers with ASD have atypically early maturation of white matter [11-14].

The presence of different patterns of connectivity abnormalities in children and adults with ASD may reflect an atypical trajectory of brain development. The early overgrowth of white and gray matter during infancy and toddlerhood in individuals with ASD is followed by 
normal or decreased growth during later childhood [15-18]. This pattern of atypical development seems to be a particular feature of the temporal, frontal, and cingulate cortices that play crucial roles in attention, emotions, and social cognition [15,18-21]. It is possible that the early increases in fMRI connectivity reported in younger children with ASD reflect their early brain overgrowth. On the other hand, the under-connectivity reported in adolescences and adults with ASD may be linked to the reduced brain growth during later childhood and/or to some compensatory processes.

Siblings of children with ASD have an increased risk of developing the disorder [22]. Therefore, studies in infant siblings of children with ASD allow one to study the early signs of the disorder before reliable diagnosis is obtained in later childhood. A recent longitudinal study found atypical developmental trajectories of cortical fiber tracts in at-risk infants who displayed ASD features at 2 years of life [14]. Specifically, development of most fiber tracts studied in the infants with later ASD was characterized by higher fractional anisotropy (FA) values at 6 months, followed by slower change over time relative to high-risk infants with few autistic traits assessed at 24 months. Thus, by 24 months of age, those with emerging ASD had lower FA values.

Structural and functional connectivity are not unequivocally associated [23]. It is therefore unclear if, and how, early differences in structural connectivity might affect emerging functional connections to result in later diagnosed ASD or, indeed, how early differences in functional connectivity might sculpt later appearing differences in structural connectivity. While in adults functional connectivity has been mainly investigated with fMRI, this method has practical limitations in infants and young children. Even in the youngest children, however, functional connectivity can be measured noninvasively with EEG. While motion inside the MRI scanner may create artifacts presenting a serious problem for analysis of connectivity [24], EEG connectivity is unlikely to be affected by motion in a similar adverse way because the EEG electrodes are in fixed locations on the scalp. In addition, the high time resolution of the method allows one to measure phase coupling of the oscillatory EEG signals indicating synchronization of neuronal populations on a millisecond timescale [25].

Although EEG has been widely used to investigate possible neural markers of ASD in adults and children, only two studies have investigated 'ongoing' EEG in infants at risk for later autism [26,27], and neither of these measured connectivity. Tierney and colleagues [27] and Bosl and colleagues [26] describe differences in quantitative EEG features between infants at high and low familial risk for ASD (i.e., infants who had an older sibling with ASD), but the actual outcome of infants 'at risk' was not known at the time of investigation.

In the present study, we used high-density EEG to examine functional connectivity in a cohort of 14month-old infants at low or high familial risk for ASD and then assessed symptoms of ASD at age 3 years. Different frequencies of EEG and MEG oscillations are associated with different cognitive, emotional, and motor processes $[25,28,29]$ and may reflect activity in different brain networks. Further, atypical EEG connectivity in neuropsychiatric disorders may be specific to particular frequency bands [30,31]. In this study, we recorded EEG while infants attended to a series of videos and then analyzed connectivity within the infant alpha band. There were several reasons for focusing on the alpha frequency band. First, the alpha rhythm is intimately related to attention processes in adults [28,32] and infants [33] and alpha-range connectivity increases during states of attentiveness $[32,34]$. Therefore, we expected the presence of reliable alpha-range connectivity during sustained attention to the videos in our study. Second, alpha activity is to a lesser extent than theta activity modulated by interindividual differences in emotional and cognitive engagement $[35,36]$. Therefore, we expected that the interindividual variations in these uncontrolled factors during passive viewing of the videos would contribute less to the alpha then into the theta frequency band. Third, the presence of alpha peak in the infant EEG power spectra suggests the presence of distinct rhythmic processes at this frequency and, correspondingly, good signal-tonoise ratio. Fourth, alpha activity is less prone to contamination by muscle artifacts than the EEG signal of higher frequencies $[37,38]$ and is less prone to contamination by movement artifacts than the delta activity. Considering all these factors, we hypothesized that in infants any altered coupling between cortical regions during attention to videos would be most reliably detected in this frequency band.

The magnitude of alpha-range connectivity can be modulated by a participant's functional state and behavior $[34,39,40]$. In order to assess the effect of ongoing behavioral state on EEG connectivity, and to control for possible outcome group differences in behavior, we complemented the EEG analysis with an analysis of infants' behavior during the EEG recording session.

Siegel et al. [25] point to several important challenges for M/EEG studies of functional brain connectivity. First, due to volume conduction, the electrical potentials generated by neuronal activity are not only measured in the direct vicinity of neuronal sources but can also be measured at distant sites, substantially limiting the interpretation of between-channel synchronization measured by, e.g., coherence of phase locking. The authors suggested that one effective strategy for addressing this confounding factor is 
to confine the analysis to noninstantaneous correlations, e.g., by looking at the phase-lagged part of coherence. In the present study, we analyzed phase-lagged connectivity using a recent method characterized by improved sensitivity [41]. Second, Siegel et al. note that differences in measured correlations can be driven by differences in the signal-to-noise ratio (SNR). Even if the true correlation between two brain sources does not differ between experimental groups, differences in the signal amplitude alone lead to differences in the SNR and thus to differences in the connectivity measures. To exclude this confounding factor, we controlled for differences in signal amplitude between the groups. Third, a large number of interactions between regions/electrodes imposes the problem of multiple comparisons. To circumvent this problem, we applied the method for statistical analysis on large networks-network-based statistics (NBS) [42].

\section{Methods}

\section{Participants and clinical assessment}

This study is a part of the British Autism Study of Infant Siblings (BASIS), a UK collaborative network facilitating research with infants at risk for autism (www. basisnetwork.org) [43]. The study involved infants at high (HR) and low (LR) familial risk for ASD. Recruitment, ethical approval (UK National Health Service National Research Ethics Service London REC 08/H0718/ 76), and informed consent, as well as background data on participating families, were made available for the current study through the BASIS. The recruitment and diagnostic procedures, as well as attrition rate, are described in Additional file 2. EEG recording was performed when infants were on average 14 months old (range 12-17 months). Sufficient artifact-free EEG was available for $28 \mathrm{HR}$ and $26 \mathrm{LR}$ participants. At 36 months, a battery of clinical research measures was administered to HR participants (see Additional file 2: Table S2 for details). From the $28 \mathrm{HR}$ infants, 10 were diagnosed with ASD at 3 years of age (HR-ASD). Demographic characteristics of the sample are given in Table 1. See Additional file 2 for details on participants' enrollment, attrition rate, and details of the diagnostic assessment. The investigators involved in EEG processing and behavioral coding were blind to the outcome of the HR participants up to the final stage of data analysis.

\section{Experimental procedure}

The infants sat on their parents' laps at a $60-\mathrm{cm}$ distance from a $40 \times 29 \mathrm{~cm}$ CRT monitor. Continuous EEG was sampled while participants watched three types of video stimuli, each lasting for 30-40 s: (1) a woman singing nursery rhymes or playing peek-a-boo ('social' video), (2) brightly colored toys moving and producing sounds ('nonsocial' video), and (3) the same sounding toys manipulated by a human hand ('nonsocial' video). Three triplets of video stimuli were presented in random order within the triplet (1-2-3, 2-3-1, etc.), but constant across the triplets for each participant. This resulted in nine 30-40-s EEG segments. Infants' behavior during EEG session was recorded with a video camera.

\section{Behavioral analysis}

Infants' behavior and events interfering with attention to the experimental stimuli were coded off-line. The data from LR and HR babies were coded in a pseudo-random order. The following parameters were coded: (1) looking at the screen; (2) gross body, head, or arm movements; (3) crying; (4) smiling; and (5) interference from a parent, experimenter, or an activity interfering with movie watching (e.g., eating, sucking a pacifier). The infant was rated as attending to the video when she/he looked at the screen, did not move, and was not distressed. To assess inter-coder reliability, videos of five randomly chosen HR and five randomly chosen LR participants were double-coded by another researcher. High reliability was obtained for all variables (Spearman rho: looking 0.89 , movements 0.85 , crying 0.98 , smiling 0.92 , attention 0.92 , interference 0.96 ).

\section{EEG acquisition and preprocessing}

EEG was recorded using a 128-electrode HydroCel Geodesic Sensor Net (EGI, Eugene, OR) with respect to the vertex and sampled at $500 \mathrm{~Hz}$. Twelve ridge electrodes most often contaminated by artifacts were excluded from

\section{Table 1 Characteristics of the sample}

\begin{tabular}{llll}
\hline & LR & HR-no-ASD & HR-ASD \\
\hline Number of male/female & $12 / 14$ & $3 / 15^{* *}$ & $7 / 3$ \\
Age at EEG, in months & $14.7(1.2)$ & $14.3(1.7)$ & $14.4(1.3)$ \\
Age at diagnostic assessment, in months & & $37.5(4.8)$ & $38.6(2.0)$ \\
Mullen Early Learning Composite, 14 months $^{\mathrm{a}}$ & $104(17)^{* *}$ & $100(12)^{*}$ & $86(15)$ \\
Mullen Early Learning Composite, 36 months $^{\mathrm{a}}$ & $115(14)^{*}$ & $110(18)$ & $100(27)$ \\
\hline
\end{tabular}

Notes: Means and standard deviations (in parentheses) are given for the ages and the Mullen ELC; difference between HR-ASD and comparison groups: ${ }^{*} P<0.05$, **P $<0.01$.

${ }^{a}$ Mullen was available for all but 1 of 26 LR infants at 14 months and 1 of 10 HR-ASD children at 36 months. 
analysis resulting in a 116-electrode layout (see Additional file 2: Figure S2). Data preprocessing and analysis was performed using FieldTrip (http://fieldtrip.fcdonders.nl/) as well as in-house software. The behavioral coding results were synchronized with EEG, and the periods when the baby was not looking at the screen, performed gross body, heard, or arm movements, or cried, as well as the periods of interference, were excluded from analysis. EEG was visually inspected for artifacts. The average length of the usable uninterrupted data periods (i.e., periods of artifact-free EEG corresponding to attentiveness in the absence of interference) did not differ between the HR-ASD and control groups (HR-ASD: 3.9 s, HR-no-ASD: 3.8 s, LR: 3.1 s; HR-ASD vs. LR and HR-ASD vs. HR-no-ASD: Mann-Whitney $U$-test $p$ 's > 0.1 ). Each of the uninterrupted data periods was then segmented into 1 -s segments with $50 \%$ overlap starting from the beginning of each clean EEG segment. The end part of the period shorter than one epoch was not analyzed. Neither number of included segments (minimum 120, see Additional file 2: Figure S1 for details) nor percent of interpolated data differed between HRASD and comparison groups (LR, HR-no-ASD; all T's < 1.0, P's > 0.33). See Additional file 2 for the preprocessing details.

\section{EEG power and connectivity analysis}

Fast Fourier transforms (FFTs) were computed for each 1-s segment after removal of the mean (baseline correction) and application of the Hanning window. Power spectra were calculated as the magnitude-squared FFTs averaged across segments.

EEG connectivity can be strongly inflated by volume conduction [44]. Newly developed methods for assessment of phase-lagged connectivity allow us to avoid this problem by analyzing phase-shifted interactions. The advantage of this approach is that the presence of a consistent, nonzero phase lag between two time series cannot be explained by volume conduction from a single strong source and therefore reveals true interactions between different underlying generators. Phase relations between time series $X$ and $Y$ can be estimated by calculating their cross-spectrum for each of $N$ data epochs. The negative value of the imaginary part (IP) of the cross-spectrum is equivalent of the signal $X$ lagging signal $Y$ in phase, while the opposite is true for the positive IP values. A few metrics have been suggested that quantify consistency of phase lag across data epochs or time points $[41,45-47]$. In the present study, we used debiased weighted phase lag index (dbWPLI) [41] (see Additional file 2 for details). The significant advantages of this method over the other phase-lagged connectivity methods include its negligibly small sampling bias and improved capacity to detect true phase synchronization
[41]. The dbWPLI values close to zero indicate absence of phase-lagged coupling while ' 1 ' corresponds to the strongest possible coupling.

\section{Statistical analysis}

Alpha power $(7-8 \mathrm{~Hz})$ was log-transformed prior to statistical analysis in order to normalize the distribution. We analyzed group differences in grand average alpha power, as well as the power of posterior alpha and central mu rhythms (see Additional file 2: Figure S2). A NBS [42] was used to compare network differences between HR-ASD and comparison groups (LR, HR-no-ASD), in order to control for the family-wise error rate (FWER) when testing is performed at each of the 6,670 connections. The primary cluster-defining threshold (Mann-Whitney $U$-test: $P<0.05$, one-tailed, $Z>1.96$ ) was first used to identify supra-threshold connections, within which the size (i.e., number of edges) of any connected components was then determined. A corrected $P$ value was calculated for each component using the null distribution of maximal connected component size, which was derived empirically using a nonparametric permutation approach (5,000 permutations, $P<0.05)$. In addition, we calculated global alpha connectivity as an average dbWPLI over all possible pairs of connections. In cases where the distributions of connectivity values were not Gaussian (Shapiro-Wilk, $P<$ $0.05)$, nonparametric tests were used to evaluate correlations and group differences.

\section{Results}

\section{Behavioral analysis}

The summary of behavioral results across the whole session, as well as separately for social and nonsocial videos (see Experimental procedure), is given in Additional file 2: Tables S3-S5. There were no significant differences between HR-ASD and the comparison groups in percent of time spent (1) looking at the videos, (2) moving, (3) watching the video without movement or negative affect (i.e., attending to videos), or (4) displaying affect (cry, smiles). To examine the possibility that infants from HR-ASD and the comparison groups preferred to attend to different types of stimuli (social vs. nonsocial), we tested for an effect of video on the infant's attention using a repeated measures ANOVA with factors Stimulus-Type and Group. No violation of ANOVA assumption about homogeneity of variance was detected (Box's $M$ statistics, Levene's test, $P>0.05)$. There was a main effect of Stimulus-Type $\left(F_{(1,53)}=91.2, P<0.0001\right)$, indicating greater attention to social than nonsocial videos (percent of time when infants attended to social stimuli: mean $=91.1$, sd $=9.6$; nonsocial: mean $=74.0$, sd $=14.6$ ), but no effect of Group $\left(F_{(2,51)}=0.36, P=0.7\right)$ or Group $\times$ StimulusType interaction $\left(F_{(2,51)}=1.3, P=0.28\right)$. 


\section{EEG alpha power}

All groups demonstrated occipital alpha and central mu spectral power peaks within infant alpha range [48], with a maximum at 7-8 $\mathrm{Hz}$. No group differences in grand average alpha power, posterior alpha power, or $\mathrm{mu}$ power were found (see Additional file 2: Figures S2-S4 for details).

\section{The dbWPLI spectrum and distribution}

In all experimental groups, the median values of global connectivity reached maximum at $7-8 \mathrm{~Hz}$ (Figure $1 \mathrm{~A}$ ), suggesting that the major part of interregional coupling during audiovisual attention occurred within the infant alpha band [48]. The other peak of grand average dbWPLI was observed in the theta range $(4-5 \mathrm{~Hz})$. The median value in the $4-5 \mathrm{~Hz}$ band (median $=0.015$, range: $0.001-0.232$ ) was lower than that in the $7-8 \mathrm{~Hz}$ band (median $=0.024$, range: 0.003-0.068). We further limited the analysis to connectivity in the 7-8 $\mathrm{Hz}$ frequency range and provided the results for the theta in Additional file 2.

The choice of the narrow 7-8-Hz alpha band was justified by a sharp drop in global connectivity at either higher or lower frequencies in all groups of subjects (Figure 1A). Importantly, this frequency range largely escapes contamination by myogenic activity from the cranial muscles, which represent a major problem for analysis of ongoing high-frequency oscillations [38]. Figure $1 \mathrm{~B}$ shows the scalp distribution of alpha dbWPLI values averaged for each electrode across all possible connections.

\section{Group differences in alpha connectivity}

For the HR-ASD vs. LR group comparison, the NBS identified one widespread pairwise cluster of hyper-connected regions in the HR-ASD group ( $N$ of nodes $=108, N$ of edges $=609, P=0.035$, Figure 2A; see also Additional file 2: Figure S5). A similar result was obtained for the HRASD vs. HR-no-ASD comparison ( $N$ of nodes $=113, N$ of edges $=841, P=0.015$, Figure $2 \mathrm{~A}$ ). When the analysis was repeated separately for the 'social' and 'nonsocial' videos, similar NBS results were obtained, suggesting that alpha hyper-connectivity occurs in infants that go on to ASD regardless of the type of video presented (see Additional file 2: Figure S6). The connections that were elevated in infants with ASD in relation to both LR and HR-no-ASD infants are plotted in Figure 2B. The electrodes with the greatest number of elevated connections were located in left fronto-central and right fronto-centro-temporal regions (Figure 2C).

The group means (LR: 0.0279, sd $=0.0157$; HR-noASD: $0.0237, \mathrm{sd}=0.0173$; HR-ASD: $0.0440 ; \mathrm{sd}=$ 0.0224 ) and individual global connectivity values are shown in Figure 1C. The non-Gaussian distribution of global connectivity values was observed in the HR-ASD (Shapiro-Wilk $W=0.82, P=0.026$ ) and HR-no-ASD (Shapiro-Wilk $W=0.82, P=0.003$ ) groups. Therefore, the nonparametric Mann-Whitney $U$-test was applied to test for group differences in global connectivity. In line with the NBS results, the global alpha connectivity was also significantly elevated in the HR-ASD infants (HR-ASD vs. LR: $U_{(10,26)}=73, Z=2.0$, exact two-tailed $P=0.045$; HR-ASD vs. HR-no-ASD: $U_{(10,18)}=48, Z=1.99$, exact two-tailed $P=0.045)$. Figure $1 C$ shows that the increase of global connectivity in the HR-ASD group was driven by six of the ten infants.

The additional analysis suggested that group differences in connectivity were not dominated by connections within the right hemisphere or left hemisphere or
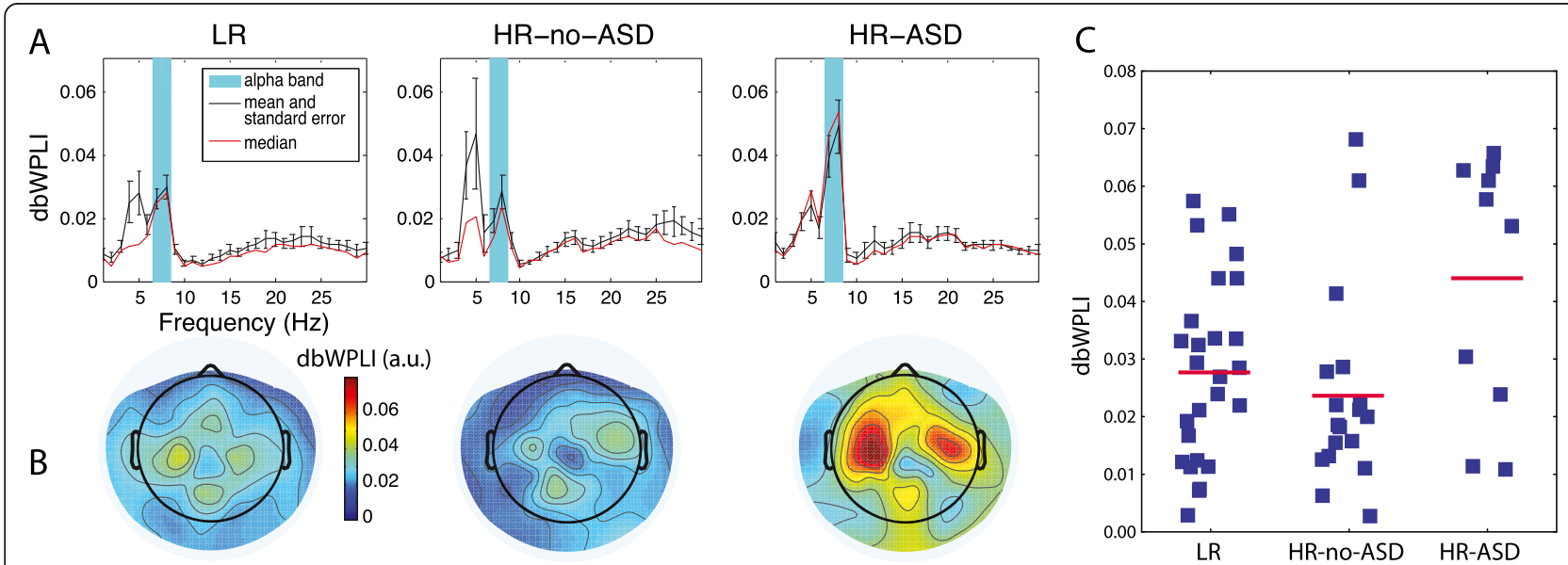

Figure 1 Phase-lagged connectivity in the three groups of participants: LR $(N=26)$, HR-no-ASD $(N=18)$, HR-ASD $(N=10)$. (A) Global connectivity as a function of frequency. Black lines show group means with vertical bars denoting standard errors; red lines show group median values. Cyan bars mark 7-8 Hz alpha range. (B) Scalp distributions of alpha-range dbWPLI values averaged for each electrode across all its connections (115 for each electrode). (C) Group means (red lines) and variability of global alpha connectivity. 
A
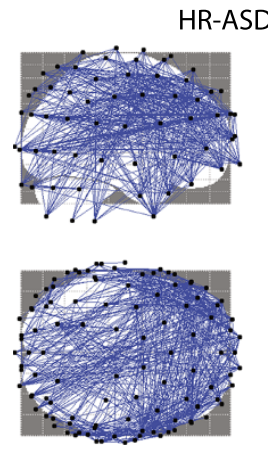

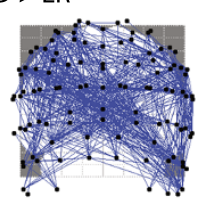

Number of

edges: 609

Number of

nodes: 108

$P=0.035$
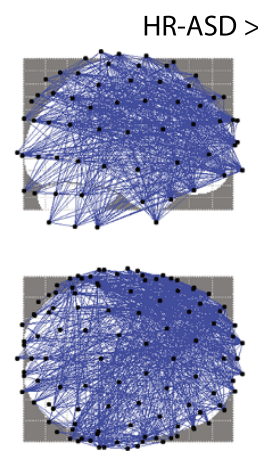

B

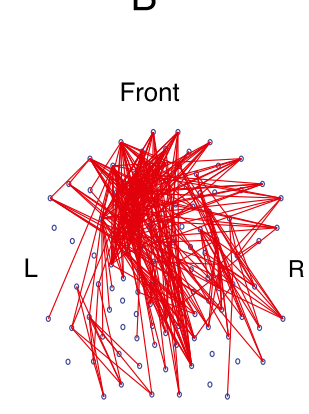

C

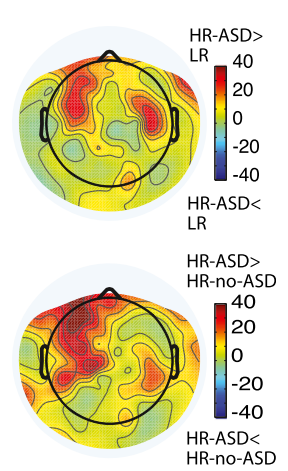

Figure 2 Group differences in alpha-range connectivity between HR-ASD $(N=10)$ and comparison groups (LR, $N=26 ; H R-n o-A S D$, $N=18$ ). (A) Networks of increased connections in infants with ASD. NBS showed significantly higher functional connectivity in HR-ASD infants as compared to both LR and HR-no-ASD infants. The nodes (electrodes) and edges of the hyper-connected networks are loosely modeled on the standard brain image. (B) Overlap of the NBS clusters of the elevated connections revealed by HR-ASD vs. LR and HR-ASD vs. HR-no-ASD comparisons. (C) Difference between numbers of connections elevated (Mann-Whitney, $P<0.05$, uncorrected) in the HR-ASD group and in comparison groups. Positive values correspond to a greater number of elevated connections in HR-ASD infants than in the comparison groups. Negative values correspond to a greater number of elevated connections in the comparison groups. Note clustering of over-connected sites over fronto-central regions.

by inter-hemispheric connections (Additional file 2: Figure S7). Moreover, the average connectivity calculated in the HR-ASD group separately for each hemisphere did not differ between hemispheres. However, there was a tendency for a higher connectivity in the left than in the right anterior area in participants with later ASD (see Additional file 2: Figure S8).

To test if the differences in connectivity between HRASD and the comparison groups could be explained by differences in gender or developmental level (Table 1), we analyzed effects of these variables separately in each experimental group. The absence of significant effects of these factors on connectivity (Additional file 2: Tables S7 and S8) makes such a possibility unlikely. There was no correlation between global connectivity and age in either of the groups (Additional file 2: Figure S10).

\section{Group differences in short- and long-range connectivity}

It has been previously suggested that children with ASD have a decreased long-to-short EEG connectivity ratio [49]. To check whether the hyper-connectivity in the HRASD group was due to short- or long-range connections, we plotted the probability of group differences in connectivity ( $Z$-scores, Mann-Whitney $U$-test) as a function of between-electrode Euclidian distance (Figure 3). Inspection of the plots indicates that increased connectivity in the HR-ASD group does not depend on scalp electrode distances (see also Additional file 2: Figure S10).
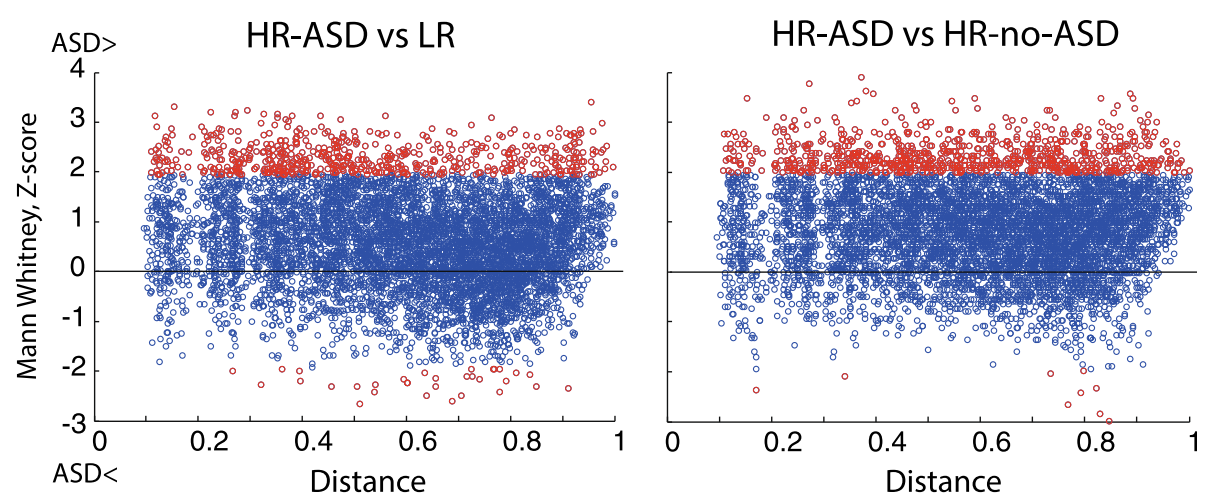

Figure 3 Probability of group differences as a function of between-electrode distance. Ordinate: Z-scores, Mann-Whitney U-test; abscissa: Euclidian distance between electrodes, where 1 corresponds to the maximal possible distance. Each pair of electrodes is represented by a dot. Red dots show $Z$ values higher or lower then $1.96(P<0.05$, two-tailed). For both HR-ASD $(N=10)$ vs. LR $(N=26)$ (left panel) and HR-ASD $(N=18)$ vs. HR-no-ASD $(N=18)$ (right panel) comparisons, the increased connectivity values in the HR-ASD group are observed irrespective of inter-electrode distance. 


\section{Alpha connectivity and severity of autism traits}

In the whole sample of HR infants, there was a trend correlation between global connectivity and the Autism Diagnostic Interview-Revised (ADI-R) Restricted and Repetitive Behaviors (RRB) scale (Spearman rho $=0.35$, $N=26, P=0.072$ ), as well as with a composite (arithmetic sum) of ADI-R Social and Communication scales (Spearman rho $=0.38, N=26, P=0.053$ ) (Figure 4, upper panel) assessed at 3 years. No significant correlations with the Autism Diagnostic Observation Schedule (ADOS) Social Communication Total score or RRB score were found ( $P$ 's $>0.35)$. We further checked if the alpha-range connectivity in the regions that were hyper-connected in the HR-ASD infants was related to the severity of autism symptoms within the HR-ASD group. To do so, we calculated Spearman's rank correlations between ADI-R/ADOS scales and dbWPLI averaged across all connections that reliably differentiated the HR-ASD from both comparison groups (Figure 2C). A significant correlation was found for the ADI-R RRB score (Spearman rho $=0.81, N=9, P=0.009$, Figure 4, lower panel), but not for the ADI Social and Communication composite score (Spearman $r h o=0.36, P=0.33$ ) or for ADOS domains ( $P$ 's $>0.35)$. No correlation with ADI-RRB has been found in the HR-no-ASD group, although this can be due to low variability of ADI-RRB scores in this group (the scores ranged 1-7 in the HR-
ASD infants, while only two infants in the HR-no-ASD group had the ADI-RRB score over 2).

\section{Discussion}

We observed increased phase-lagged alpha EEG connectivity in a group of 14-month-old infants who were later diagnosed with ASD at 3 years of age. The elevated connectivity was present despite the lack of measurable differences in behavior during the EEG data collection or differences in EEG spectral power. Further, in participants with ASD, this measure at 14 months correlated with the severity of restricted and repetitive behaviors at 3 years.

Studies in adults show a correlation between alpha EEG coherence and the structural integrity of white matter [50]. The high alpha-range connectivity in HR-ASD infants in our study may reflect the early maturation of white matter tracts previously reported in toddlers and young children with ASD [11-14]. The increases in white matter in ASD appear to reduce in toddlerhood [14] or later childhood [51,52], finally resulting in predominantly hypo-connectivity between cortical areas in adults with ASD [7]. These age-related differences in white matter integrity may potentially explain the fact that the alpharange hyper-connectivity detected in infants who later go on to have ASD in our study is later followed by alpharange hypo-connectivity in adolescence and adulthood [31,39,53-56].

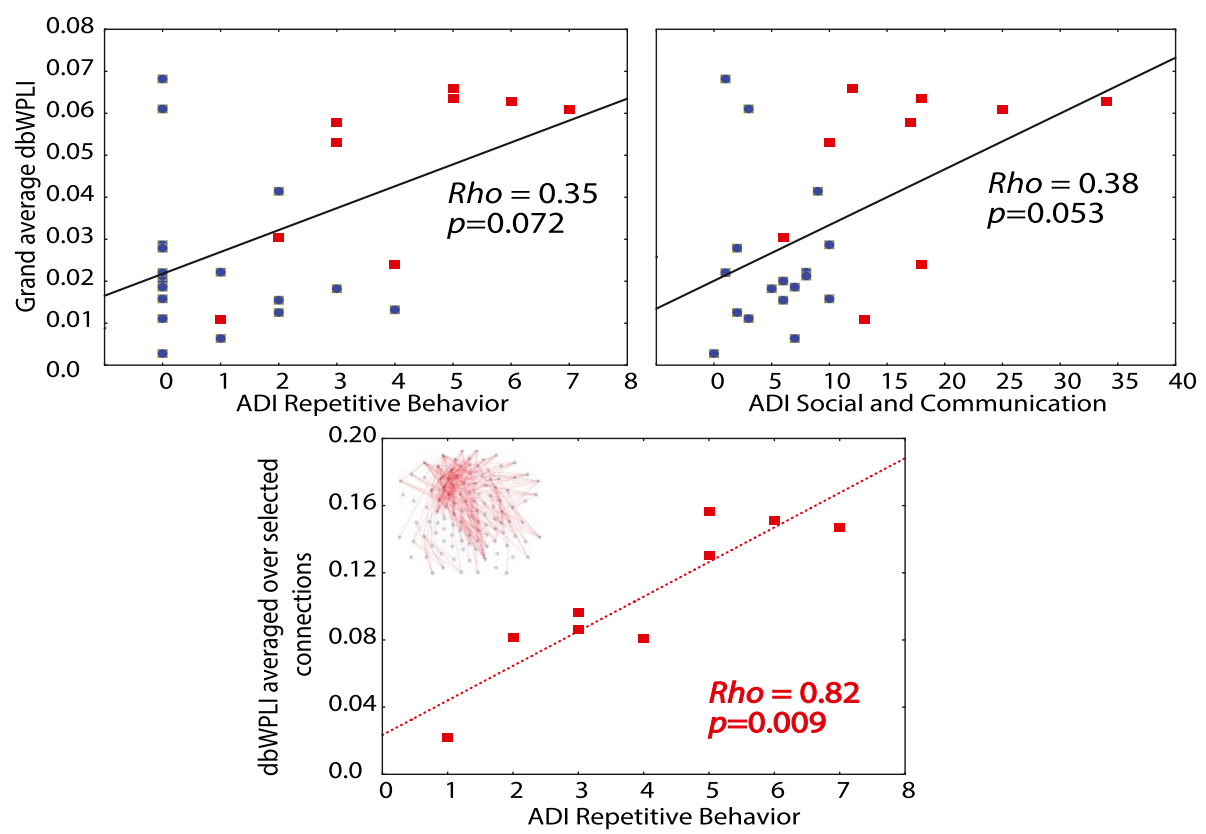

Figure 4 Correlations (Spearman rho) between alpha-range connectivity and ADI-R domains in HR-ASD infants $\left(N=9^{*}\right)$ and combined HR group $\left(N=26^{*}\right)$. Red and blue squares mark, respectively, HR-ASD and HR-no-ASD infants. Global connectivity (upper panels) marginally correlates with the ADI-R Social and Communication composite score and with the ADI Repetitive Behavior score in the combined HR sample. The lower panel shows the correlation between the ADI-R Repetitive Behavior score and dbWPLI values averaged across connections that were significantly elevated in HR-ASD comparative to both comparison groups. *Note that one HR-ASD infant and one HR-no-ASD infant did not have ADI-R data. 
It is also possible that the atypical connectivity observed in ASD is a sequela of an altered excitation/inhibition (E/ I) ratio [57]. The number of parvalbumin-expressing inhibitory interneurons [58], as well as GABA receptors and the receptors' benzodiazepine binding sites [59], is reduced in autism, suggesting reduced cortical inhibition. In typical individuals, the glutamate/GABA ratio correlates positively with functional connectivity in the default mode network [60]. It is therefore possible that the elevated connectivity that we observed in HR-ASD infants results from their elevated E/I ratio. The later decrease in connectivity in adolescents and adults with ASD may then reflect a compensatory reaction to reduce cortical excitability. The future studies may help to investigate the putative link between abnormal neural connectivity and elevated E/I ratio in ASD by looking at connectivity measures in relation to ongoing and event-related gamma oscillations [61,62].

The increased alpha-range connectivity in HR-ASD infants was widespread across the scalp (Figures $2 \mathrm{~A}$ and 3 ), a finding that is in accord with DTI evidence on the presence of atypical development trajectories for white matter in all of the major tracts studied in toddlers with high autism traits [14] and with recent fMRI findings in children with ASD [8]. Nevertheless, clustering of significant dbWPLI differences over the anterior, and particularly left frontal (Figure 2B, C, see also Additional file 2: Figure S8), regions implies some degree of regional specificity in the atypical functional connectivity and suggests that the left frontal cortex may be particularly strongly over-connected in some infants who later go on to an ASD diagnosis. Prominent developmental atypicalities have previously been reported in the frontal lobes of children and adults with ASD [15,16,63]. Interestingly, Bashat and colleagues [11,13] found abnormally mature white matter predominantly in the left frontal lobe in preschool children with ASD.

Alpha-range connectivity in infants who went on to ASD correlated with their later severity of RRB (Figure 4, lower panel). This finding suggests that alpha hyperconnectivity may reflect an important aspect of atypical brain function in many cases of emerging ASD. The link between hyper-connectivity and severity of RRB has previously been reported in resting-state fMRI studies. Uddin et al. [9] observed that hyper-connectivity in the 'salience network' predicted greater incidence of RRB in 10-yearold children with ASD. Delmonte et al. [64] observed a link between the severity of RRB and elevated frontostriatal connectivity in adolescents with ASD. Interestingly, correlations between structural changes in the striatum and repetitive behaviors have been previously reported $[65,66]$ and may be secondary to changes in frontal areas or fronto-striatal connectivity [65]. In adults with ASD, the higher functional connectivity between left dorsal ACC and the frontal eye field was associated with more severe
$\mathrm{RRB}$, even though connectivity between these structures was reduced in the ASD participants relative to neurotypical controls [67]. A recent morphological study of shortrange intrinsic cortico-cortical connections also reports an association between frontal connectivity and the ADI-R RRB score in adults with ASD [68]. Specifically, the decreased 'wiring cost' (and thus potentially increased cortico-cortical connectivity) in several frontal regions in participants with ASD correlated with their tendency to engage in repetitive behaviors. Although the sources of the elevated alpha connectivity in our study cannot be localized precisely based exclusively on surface EEG, our results accord well with the previous studies implicating frontal hyper-connectivity in repetitive behaviors in ASD.

It should be noted that reduced connectivity in young children with ASD has also been previously reported [69]. Using fMRI, Dinstein et al. [69] found that toddlers with ASD had reduced synchronization between corresponding 'language areas' of the two hemispheres during sleep. Moreover, the reduced functional connectivity correlated with poorer language abilities and with greater social and communication deficits. Further studies are therefore needed to investigate in ASD possible differences in functional connectivity measured at faster (EEG phase lag) and slower (fMRI) timescales, as well as modulations of connectivity by participant's age and functional state.

It has been suggested that in adults with ASD, local hyper-connectivity in the frontal cortex occurs together with long-range hypo-connectivity $[7,16]$. We have found elevated connectivity in infants with later ASD irrespective of between-electrode distance (Figure 3). Notably, the phase-lagged connectivity assessed in the present study may underestimate short-range connections as they are more likely than the long-range connections to occur without measurable phase lag. Other methods, such as, for example, alpha to gamma phase-amplitude coupling, may provide better estimates of short-range connectivity in ASD [54].

Although no EEG connectivity studies have been done so far in infants with later diagnosis of ASD, such studies have been previously performed in diagnosed children and adults. The studies in adults and adolescents with ASD mainly reported decreased MEG and EEG connectivity in the alpha frequency range [31,39,53-56], although negative findings have also been reported [70,71]. Studies in children produced more variable results, with findings of decreased [72,73], not changed [49,74], both decreased and increased [75], or increased [76] alpharange connectivity in ASD. Apart from differences in experimental samples, there are other factors that might potentially contribute to the findings. Among these are the choice of frequency band and reference electrode for EEG recording (e.g., Common Mode Sense-Driven Right 
Leg reference [74], linked earlobes [73,76], Laplacian [75], or average reference [72]), presence of group differences in alpha power (and SNR) [72,76] or lack of information on such differences $[49,74,75]$, and differences in experimental paradigms, such as undefined state [49], rest with eyes closed [73], photo-driving [76], or visual stimulation with long (seconds) intervals [72,74]. Some of these factors could influence group differences in alpha-range connectivity in our study. Moreover, the majority of the previous studies in children assessed functional connectivity using coherence-the measure that is sensitive to volume conduction. The application of the phaselagged connectivity in our study should favor detection of the 'true' functional connectivity differences that could otherwise have been altered or canceled out by volume conduction effects that might be stronger in individuals possessing greater alpha power.

Although EEG and MEG have been previously used to study functional connectivity in ASD (see Additional file 1 for review), only one study, similarly to our present study, analyzed phase-lagged connectivity [74]. Boersma and colleagues recorded EEG in children (mean age 3 years) during presentation of visual stimuli but did not find group differences in low-frequency $(4-10 \mathrm{~Hz})$ connectivity. One possibility is that the elevated alpha range connectivity in ASD is more prominent during infancy and then decreases to the third year of life. Another possibility is that connectivity differences can still be detected in 3-year-olds with ASD but in narrower ageappropriate frequency bands and/or with a greater temporal quantity of data.

Figure $2 \mathrm{C}$ suggests that not all participants who were later diagnosed with ASD in our study had increased alpha-range connectivity as infants. Alterations in functional brain connectivity in ASD may depend on the presence of a risk allele of a particular 'connectivitycrucial' gene $[77,78]$ or on a combination of genes unique to an individual. Possibly, the atypical connectivity patterns characterize only a subset of the HR-ASD infants with such genetic predisposition. Our findings indicate that hyper-connectivity during infancy may be a feature of participants who later go on to show higher levels of restrictive and repetitive behavior (Figure 4). Considering the small group size of the HR-ASD infants in our study, further studies with larger samples are needed to elucidate behavioral features that may differentiate children with and without early alpha-range hyper-connectivity.

\section{Conclusions}

In summary, our study provides the first EEG evidence of functional brain hyper-connectivity in infants who later go on to ASD. The hyper-connectivity may reflect morphological white matter abnormalities previously found in infants, toddlers, and young children with ASD and/or an elevated neural excitation/inhibition ratio. Future studies with participants of wider age ranges using robust EEG connectivity measures in combination with other neuroimaging techniques will help understand better the developmental course of neural connectivity in ASD and help provide neurophysiological biomarkers of these disorders.

\section{Additional files}

\begin{abstract}
Additional file 1: Short summary of the previous EEG and MEG studies of brain functional connectivity in ASD. The file contains a table summarizing the main results of the studies and the methods used in these studies.

Additional file 2: Supplementary methods and results. Supporting methods: participants and clinical assessment; EEG recording and preprocessing; EEG connectivity analysis; minimal number of artifact-free EEG epochs included into analysis. Supporting results and discussion: behavioral analysis; theta band findings; power analysis of alpha activity; group differences in alpha ubPLI (combined conditions): NBS results; group differences in alpha dbWPLI in social and non-social conditions: NBS results; lateralization of alpha connectivity measures; effect of gender and developmental level; global alpha connectivity and age; dbWPLI group differences in short- and long-range connections in the alpha band. Supporting references.
\end{abstract}

\section{Abbreviations}

ASD: autism spectrum disorder (s); dbWPLI: debiased weighted phase lag index; EEG: electroencephalography; FFT: fast Fourier transform; HR-ASD: high-risk participants with ASD outcome; HR: high risk; HR-no-ASD: high-risk participants with 'no ASD' outcome; LR: low risk; RRB: restricted and repetitive behaviors.

\section{Competing interests}

The authors declare that they have no competing interests.

\section{Authors' contributions}

$M E, G D, T C$, and MJ designed and conceived the experiment. ME and the BASIS Team collected the data. EO and the BASIS Team cleaned and analyzed the data. EO, EJ, GD, TC, and MJ contributed to the interpretation and writing. TC and the BASIS Team contributed to the clinical assessment and cohort characterization. All authors read and approved the final manuscript.

\section{Acknowledgements}

We are very grateful for the enormous contributions BASIS families have made towards this study. This work was supported by the UK Medical Research Council (G0701484), Autism Speaks (\#1292), the BASIS funding consortium led by Autistica (www.basisnetwork.org), and EU-AIMS, the Innovative Medicines Initiative Joint Undertaking under grant agreement no. 115300, resources of which are composed of financial contribution from the European Union's Seventh Framework Programme (FP7/2007-2013) and EFPIA companies' in-kind contribution. The BASIS Team authors in alphabetical order: Simon Baron-Cohen, Rachael Bedford, Patrick Bolton, Janice Fernandes, Natasa Ganea, Holly Garwood, Teodora Gliga, Kristelle Hudry, Michael Murias, Greg Pasco, Helena Ribeiro, Leslie Tucker, Agnes Volein, Sara Jane Webb.

\section{Author details}

${ }^{1}$ Centre for Brain and Cognitive Development, School of Psychology, Birkbeck, University of London, Henry Welcome Building, London WC1E 7HX, UK. ${ }^{2}$ Department of Psychiatry, McGill University, Montreal PQ H3A 1A1, Canada. ${ }^{3}$ Department of Psychiatry and Behavioral Sciences, School of Medicine, Duke University, Durham, NC 27705, USA. ${ }^{4}$ Department of Psychology, Institute of Psychiatry, Psychology \& Neuroscience, King's College London, London SE5 8AF, UK.

Received: 23 May 2014 Accepted: 17 October 2014

Published: 7 November 2014 


\section{References}

1. Association AP: Diagnostic and Statistical Manual of Mental Disorders 5th Edition - Text Revision (DSM-5). Washington, DC: American Psychiatric Association; 2013.

2. Delorme R, Ey E, Toro R, Leboyer M, Gillberg C, Bourgeron T: Progress toward treatments for synaptic defects in autism. Nat Med 2013, 19:685-694

3. Parikshak NN, Luo R, Zhang A, Won H, Lowe JK, Chandran V, Horvath S, Geschwind DH: Integrative functional genomic analyses implicate specific molecular pathways and circuits in autism. Cell 2013, 155:1008-1021.

4. Johnson $\mathrm{MH}$ : Interactive specialization: a domain-general framework for human functional brain development? Dev Cogn Neurosci 2011, 1:7-21.

5. Dawson G: Early behavioral intervention, brain plasticity, and the prevention of autism spectrum disorder. Dev Psychopathol 2008, 20:775-803.

6. Just MA, Cherkassky VL, Keller TA, Minshew NJ: Cortical activation and synchronization during sentence comprehension in high-functioning autism: evidence of underconnectivity. Brain 2004, 127:1811-1821.

7. Vissers ME, Cohen MX, Geurts HM: Brain connectivity and high functioning autism: a promising path of research that needs refined models, methodological convergence, and stronger behavioral links. Neurosci Biobehav Rev 2012, 36:604-625.

8. Supekar K, Uddin LQ, Khouzam A, Phillips J, Gaillard WD, Kenworthy LE, Yerys BE, Vaidya CJ, Menon V: Brain hyperconnectivity in children with autism and its links to social deficits. Cell Rep 2013, 5:738-747.

9. Uddin LQ, Supekar K, Lynch CJ, Khouzam A, Phillips J, Feinstein C, Ryali S, Menon V: Salience network-based classification and prediction of symptom severity in children with autism. JAMA Psychiatry 2013, 70:869-879.

10. Uddin LQ, Supekar K, Menon V: Reconceptualizing functional brain connectivity in autism from a developmental perspective. Front Hum Neurosci 2013, 7:458.

11. Bashat DB, Kronfeld-Duenias V, Zachor DA, Ekstein PM, Hendler T, Tarrasch R, Even A, Levy Y, Sira LB: Accelerated maturation of white matter in young children with autism: a high b value DWI study. Neuroimage 2007, 37:40-47.

12. Billeci L, Calderoni S, Tosetti M, Catani M, Muratori F: White matte connectivity in children with autism spectrum disorders: a tract-based spatial statistics study. BMC Neurol 2012, 12:148.

13. Weinstein M, Ben-Sira L, Levy Y, Zachor DA, Ben Itzhak E, Artzi M, Tarrasch R, Eksteine PM, Hendler T, Ben Bashat D: Abnormal white matter integrity in young children with autism. Hum Brain Mapp 2011, 32:534-543.

14. Wolff JJ, Gu HB, Gerig G, Elison JT, Styner M, Gouttard S, Botteron KN, Dager SR, Dawson G, Estes AM, Evans AC, Hazlett HC, Kostopoulos P, McKinstry RC, Paterson SJ, Schultz RT, Zwaigenbaum L, Piven J: Differences in white matter fiber tract development present from 6 to 24 months in infants with autism. Am J Psychiatry 2012, 169:589-600.

15. Courchesne E, Campbell K, Solso S: Brain growth across the life span in autism: age-specific changes in anatomical pathology. Brain Res 2011, 1380:138-145

16. Courchesne $E$, Pierce $K$ : Why the frontal cortex in autism might be talking only to itself: local over-connectivity but long-distance disconnection. Curr Opin Neurobiol 2005, 15:225-230.

17. Hardan AY, Libove RA, Keshavan MS, Melhem NM, Minshew NJ: A preliminary longitudinal magnetic resonance imaging study of brain volume and cortical thickness in autism. Biol Psychiatry 2009, 66:320-326.

18. Schumann CM, Bloss CS, Barnes CC, Wideman GM, Carper RA, Akshoomoff N, Pierce K, Hagler D, Schork N, Lord C, Courchesne E: Longitudinal magnetic resonance imaging study of cortical development through early childhood in autism. J Neurosci 2010, 30:4419-4427.

19. Hazlett HC, Poe MD, Gerig G, Styner M, Chappell C, Smith RG, Vachet C, Piven J: Early brain overgrowth in autism associated with an increase in cortical surface area before age 2 years. Arch Gen Psychiatry 2011, 68:467-476.

20. Carper RA, Courchesne E: Localized enlargement of the frontal cortex in early autism. Biol Psychiatry 2005, 57:126-133.

21. Carper RA, Moses P, Tique ZD, Courchesne E: Cerebral lobes in autism: early hyperplasia and abnormal age effects. Neuroimage 2002, 16:1038-1051.

22. Lauritsen MB, Pedersen CB, Mortensen PB: Effects of familial risk factors and place of birth on the risk of autism: a nationwide register-based study. J Child Psychol Psychiatry 2005, 46:963-971.
23. Uddin LQ: Complex relationships between structural and functional brain connectivity. Trends Cogn Sci 2013, 17:24094797.

24. Power JD, Barnes KA, Snyder AZ, Schlaggar BL, Petersen SE: Spurious but systematic correlations in functional connectivity MRI networks arise from subject motion. Neuroimage 2012, 59:2142-2154

25. Siegel $M$, Donner $T H$, Engel AK: Spectral fingerprints of large-scale neuronal interactions. Nat Rev Neurosci 2012, 13:121-134.

26. BosI W, Tierney A, Tager-Flusberg H, Nelson C: EEG complexity as a biomarker for autism spectrum disorder risk. BMC Med 2011, 9:18.

27. Tierney AL, Gabard-Durnam L, Vogel-Farley V, Tager-Flusberg H, Nelson CA: Developmental trajectories of resting EEG power: an endophenotype of autism spectrum disorder. PLos One 2012, 7:e39127.

28. Klimesch W, Sauseng P, HansImayr S: EEG alpha oscillations: the inhibitiontiming hypothesis. Brain Res Rev 2007, 53:63-88

29. Ward LM: Synchronous neural oscillations and cognitive processes. Trends Cogn Sci 2003, 7:553-559.

30. Canuet L, Tellado I, Couceiro V, Fraile C, Fernandez-Novoa L, Ishii R, Takeda $M$, Cacabelos R: Resting-state network disruption and APOE genotype in Alzheimer's disease: a lagged functional connectivity study. PLos One 2012, 7:e46289.

31. Murias M, Webb SJ, Greenson J, Dawson G: Resting state cortical connectivity reflected in EEG coherence in individuals with autism. Biol Psychiatry 2007, 62:270-273.

32. Palva S, Palva JM: New vistas for alpha-frequency band oscillations. Trends Neurosci 2007, 30:150-158.

33. Orekhova EV, Stroganova TA, Posikera IN: Alpha activity as an index of cortical inhibition during sustained internally controlled attention in infants. Clin Neurophysiol 2001, 112:740-749.

34. Sauseng P, Klimesch W, Stadler W, Schabus M, Doppelmayr M, HansImayr S, Gruber WR, Birbaumer N: A shift of visual spatial attention is selectively associated with human EEG alpha activity. Eur J Neurosci 2005, 22:2917-2926.

35. Orekhova EV, Stroganova TA, Posikera IN, Elam M: EEG theta rhythm in infants and preschool children. Clin Neurophysiol 2006, 117:1047-1062.

36. Stroganova TA, Orekhova EV: EEG and infant states. In Infant EEG and Event-Related Potentials. Edited by De Haan M. Hove: Psychology Press; 2007:251-280

37. Goncharova II, McFarland DJ, Vaughan TM, Wolpaw JR: EMG contamination of EEG: spectral and topographical characteristics. Clin Neurophysiol 2003, 114:1580-1593.

38. Whithain EM, Pope KJ, Fitzgibbon SP, Lewis T, Clark CR, Loveless S, Broberg M, Wallace A, DeLosAngeles D, Lillie P, Hardy A, Fronsko R, Pulbrook A, Willoughby JO: Scalp electrical recording during paralysis: quantitative evidence that EEG frequencies above $20 \mathrm{~Hz}$ are contaminated by EMG. Clin Neurophysiol 2007, 118:1877-1888.

39. Kenet T, Orekhova EV, Bharadwaj H, Shetty NR, Israeli E, Lee AKC, Agam Y, Elam M, Joseph RM, Hamalainen MS, Manoach DS: Disconnectivity of the cortical ocular motor control network in autism spectrum disorders. Neuroimage 2012, 61:1226-1234.

40. Mima T, Oluwatimilehin T, Hiraoka T, Hallett M: Transient interhemispheric neuronal synchrony correlates with object recognition. J Neurosci 2001, 21:3942-3948

41. Vinck M, Oostenveld R, van Wingerden M, Battaglia F, Pennartz CMA: An improved index of phase-synchronization for electrophysiological data in the presence of volume-conduction, noise and sample-size bias. Neuroimage 2011, 55:1548-1565.

42. Zalesky A, Fornito A, Bullmore ET: Network-based statistic: identifying differences in brain networks. Neuroimage 2010, 53:1197-1207.

43. Elsabbagh M, Mercure E, Hudry K, Chandler S, Pasco G, Charman T, Pickles A, Baron-Cohen S, Bolton P, Johnson MH, BASIS Team: Infant neural sensitivity to dynamic eye gaze is associated with later emerging autism. Curr Biol 2012, 22:338-342.

44. Nunez PL, Silberstein RB, Shi Z, Carpenter MR, Srinivasan R, Tucker DM, Doran SM, Cadusch PJ, Wijesinghe RS: EEG coherency II: experimental comparisons of multiple measures. Clin Neurophysiol 1999, 110:469-486.

45. Nolte G, Bai O, Wheaton L, Mari Z, Vorbach S, Hallett M: Identifying true brain interaction from EEG data using the imaginary part of coherency. Clin Neurophysiol 2004, 115:2292-2307.

46. Nolte G, Ziehe A, Nikulin W, Schlogl A, Kramer N, Brismar T, Muller KR: Robustly estimating the flow direction of information in complex physical systems. Phys Rev Lett 2008, 100:234101. 
47. Stam CJ, Nolte G, Daffertshofer A: Phase lag index: assessment of functional connectivity from multi channel EEG and MEG with diminished bias from common sources. Hum Brain Mapp 2007, 28:1178-1193.

48. Stroganova TA, Orekhova EV, Posikera IN: EEG alpha rhythm in infants. Clin Neurophysio/ 1999, 110:997-1012.

49. Peters JM, Taquet M, Vega C, Jeste SS, Fernandez IS, Tan J, Nelson CA, Sahin $M$, Warfield SK: Brain functional networks in syndromic and nonsyndromic autism: a graph theoretical study of EEG connectivity. BMC Med 2013, 11:54.

50. Teipel SJ, Pogarell O, Meindl T, Dietrich O, Sydykova D, Hunklinger U, Georgii B, Mulert C, Reiser MF, Moller HJ, Hampel H: Regional networks underlying interhemispheric connectivity: an EEG and DTI study in healthy ageing and amnestic mild cognitive impairment. Hum Brain Mapp 2009, 30:2098-2119.

51. Mak-Fan KM, Morris D, Vidal J, Anagnostou E, Roberts W, Taylor MJ: White matter and development in children with an autism spectrum disorder. Autism 2013, 17:541-557.

52. Walker L, Gozzi M, Lenroot R, Thurm A, Behseta B, Swedo S, Pierpaoli C: Diffusion tensor imaging in young children with autism: biological effects and potential confounds. Biol Psychiatry 2012, 72:1043-1051.

53. Catarino A, Andrade A, Churches O, Wagner AP, Baron-Cohen S, Ring H: Task-related functional connectivity in autism spectrum conditions: an EEG study using wavelet transform coherence. Mol Autism 2013, 4:1.

54. Khan S, Gramfort A, Shetty NR, Kitzbichler MG, Ganesan S, Moran JM, Lee SM, Gabrieli JD, Tager-Flusberg HB, Joseph RM, Herbert MR, Hämäläinen MS, Kenet T: Local and long-range functional connectivity is reduced in concert in autism spectrum disorders. Proc Natl Acad Sci U S A 2013, 110:3107-3112.

55. Lazar AS, Lazar Zl, Biro A, Gyori M, Tarnok Z, Prekop C, Keszei A, Stefanik K, Gadoros J, Halasz P, Bodizs R: Reduced fronto-cortical brain connectivity during NREM sleep in Asperger syndrome: an EEG spectral and phase coherence study. Clin Neurophysiol 2010, 121:1844-1854.

56. Tsiaras V, Simos PG, Rezaie R, Sheth BR, Garyfallidis E, Castillo EM, Papanicolaou AC: Extracting biomarkers of autism from MEG resting-state functional connectivity networks. Comput Biol Med 2011, 41:1166-1177.

57. Zikopoulos B, Barbas $\mathrm{H}$ : Altered neural connectivity in excitatory and inhibitory cortical circuits in autism. Front Hum Neurosci 2013, 7:609.

58. Oblak AL, Rosene DL, Kemper TL, Bauman ML, Blatt GJ: Altered posterior cingulate cortical cyctoarchitecture, but normal density of neurons and interneurons in the posterior cingulate cortex and fusiform gyrus in autism. Autism Res 2011, 4:200-211.

59. Oblak AL, Gibbs TT, Blatt GJ: Reduced GABAA receptors and benzodiazepine binding sites in the posterior cingulate cortex and fusiform gyrus in autism. Brain Res 2011, 1380:218-228.

60. Kapogiannis D, Reiter DA, Willette AA, Mattson MP: Posteromedial cortex glutamate and GABA predict intrinsic functional connectivity of the default mode network. Neuroimage 2013, 64:112-119.

61. Yizhar O, Fenno LE, Prigge M, Schneider F, Davidson TJ, O'Shea DJ, Sohal VS, Goshen I, Finkelstein J, Paz JT, Stehfest K, Fudim R, Ramakrishnan C, Huguenard JR, Hegemann P, Deisseroth K: Neocortical excitation/inhibition balance in information processing and social dysfunction. Nature 2011, 477:171-178.

62. Saunders JA, Gandal MJ, Roberts TP, Siegel SJ: NMDA antagonist MK801 recreates auditory electrophysiology disruption present in autism and other neurodevelopmental disorders. Behav Brain Res 2012, 234:233-237.

63. Zikopoulos B, Barbas $\mathrm{H}$ : Changes in prefrontal axons may disrupt the network in autism. J Neurosci 2010, 30:14595-14609.

64. Delmonte S, Gallagher L, O'Hanlon E, McGrath J, Balsters JH: Functional and structural connectivity of frontostriatal circuitry in autism spectrum disorder. Front Hum Neurosci 2013, 7:430.

65. Langen M, Bos D, Noordermeer SD, Nederveen $H$, Van Engeland H, Durston $\mathrm{S}$ : Changes in the development of striatum are involved in repetitive behavior in autism. Biol Psychiatry 2013, 76:405-411.

66. Rojas DC, Peterson E, Winterrowd E, Reite ML, Rogers SJ, Tregellas JR: Regional gray matter volumetric changes in autism associated with social and repetitive behavior symptoms. BMC Psychiatry 2006, 6:56.

67. Agam Y, Joseph RM, Barton JJS, Manoach DS: Reduced cognitive control of response inhibition by the anterior cingulate cortex in autism spectrum disorders. Neuroimage 2010, 52:336-347.
68. Ecker C, Ronan L, Feng Y, Daly E, Murphy C, Ginestet CE, Brammer M, Fletcher PC, Bullmore ET, Suckling J, Baron-Cohen S, Williams S, Loth E, MRC AIMS Consortium, Murphy DG: Intrinsic gray-matter connectivity of the brain in adults with autism spectrum disorder. Proc Natl Acad Sci U S A 2013, 110:13222-13227.

69. Dinstein I, Pierce K, Eyler L, Solso S, Malach R, Behrmann M, Courchesne E: Disrupted neural synchronization in toddlers with autism. Neuron 2011, 70:1218-1225.

70. Leveille C, Barbeau EB, Bolduc C, Limoges E, Berthiaume C, Chevrier E, Mottron L, Godbout R: Enhanced connectivity between visual cortex and other regions of the brain in autism: a REM sleep EEG coherence study. Autism Res 2010, 3:280-285.

71. Mathewson KJ, Jetha MK, Drmic IE, Bryson SE, Goldberg JO, Schmidt LA: Regional EEG alpha power, coherence, and behavioral symptomatology in autism spectrum disorder. Clin Neurophysiol 2012, 123:1798-1809.

72. Isler JR, Martien KM, Grieve PG, Stark RI, Herbert MR: Reduced functional connectivity in visual evoked potentials in children with autism spectrum disorder. Clin Neurophysiol 2010, 121:2035-2043.

73. Coben R, Clarke AR, Hudspeth W, Barry RJ: EEG power and coherence in autistic spectrum disorder. Clin Neurophysiol 2008, 119:1002-1009.

74. Boersma M, Kemner C, de Reus MA, Collin G, Snijders TM, Hofman D, Buitelaar JK, Stam CJ, van den Heuvel MP: Disrupted functional brain networks in autistic toddlers. Brain Connect 2013, 3:41-49.

75. Duffy FH, Als H: A stable pattern of EEG spectral coherence distinguishes children with autism from neuro-typical controls - a large case control study. BMC Med 2012, 10:64.

76. Lazarev W, Pontes A, Mitrofanov AA, DeAzevedo LC: Interhemispheric asymmetry in EEG photic driving coherence in childhood autism. Clin Neurophysiol 2010, 121:145-152.

77. Rudie JD, Hernandez LM, Brown JA, Beck-Pancer D, Colich NL, Gorrindo P Thompson PM, Geschwind DH, Bookheimer SY, Levitt P, Dapretto M: Autism-associated promoter variant in MET impacts functional and structural brain networks. Neuron 2012, 75:904-915.

78. Scott-Van Zeeland AA, Abrahams BS, Alvarez-Retuerto Al, Sonnenblick LI, Rudie JD, Ghahremani D, Mumford JA, Poldrack RA, Dapretto M, Geschwind $\mathrm{DH}$, Bookheimer SY: Altered functional connectivity in frontal lobe circuits is associated with variation in the autism risk gene CNTNAP2. Sci Transl Med 2010, 2:56ra80.

doi:10.1186/1866-1955-6-40

Cite this article as: Orekhova et al.: EEG hyper-connectivity in high-risk infants is associated with later autism. Journal of Neurodevelopmental Disorders 2014 6:40.

\section{Submit your next manuscript to BioMed Central and take full advantage of:}

- Convenient online submission

- Thorough peer review

- No space constraints or color figure charges

- Immediate publication on acceptance

- Inclusion in PubMed, CAS, Scopus and Google Scholar

- Research which is freely available for redistribution 